\title{
Partial cross sections and angular distributions of resonant and nonresonant valence photoemission of $\mathbf{C}_{60}$
}

\author{
Sanja Korica, ${ }^{1}$ Daniel Rolles, ${ }^{1}$ Axel Reinköster, ${ }^{1}$ Burkhard Langer, ${ }^{2}$ Jens Viefhaus, ${ }^{1}$ Slobodan Cvejanović, ${ }^{1, *}$ and \\ Uwe Becker ${ }^{1, \uparrow}$ \\ ${ }^{1}$ Fritz-Haber-Institut der Max-Planck-Gesellschaft, Faradayweg 4-6, D-14195 Berlin, Germany \\ ${ }^{2}$ Max-Born-Institut, Max-Born-Strasse 2A, D-12489 Berlin, Germany
}

(Received 16 July 2004; published 19 January 2005)

\begin{abstract}
We have performed high-resolution measurements of photoelectrons emitted from the valence shell of $\mathrm{C}_{60}$, for both gas phase and solid state, in order to obtain branching ratios, partial cross sections, and the angular distribution anisotropy parameters of the two highest occupied molecular orbitals. The analysis is based on the Fourier transform of the cross-section oscillations and the results are corroborated by different theoretical models. In contrast to this good overall agreement between theory and experiment there is a striking disagreement with respect to predicted discrete resonance structures in the partial cross sections. Possible reasons for this behavior are discussed.
\end{abstract}

DOI: $10.1103 /$ PhysRevA.71.013203

PACS number(s): $36.40 . \mathrm{Cg}, 33.60 . \mathrm{Cv}, 33.80 . \mathrm{Eh}$

\section{INTRODUCTION}

After the discovery of $\mathrm{C}_{60}$ in 1985 [1] and the success of Krätschmer and co-workers [2] in synthesizing macroscopic quantities of this new form of pure solid carbon, its basic properties were soon studied by nearly all available techniques. Due to its unique molecular structure like a spherical shell, $\mathrm{C}_{60}$ has an interesting electronic structure which has been extensively investigated by a large number of authors, e.g., Refs. [3-7]. In the present study, the photon energy dependence of the photoelectron spectra of $\mathrm{C}_{60}$ was recorded in the range of $h \nu=20-70 \mathrm{eV}$, for both gas phase and solid state, in order to investigate the photoionization cross-section behavior of the two highest occupied molecular orbitals HOMO and HOMO-1. These photolines exhibit characteristic energy-dependent intensity oscillations that are analyzed in terms of the geometrical properties of both the cage structure and the distribution of the delocalized electron cloud of the highest occupied molecular orbitals. The overall agreement between theory and experiment, concerning the general shape of the cross-section behavior, is very good. The angular distribution asymmetry parameter $\beta$, measured during the same experiment, shows no pronounced oscillations, rather rising continuously at higher photon energies. This is a fullerene-unspecific behavior, also known from other carboncontaining molecules, such as $\mathrm{CO}$ [8].

\section{EXPERIMENT}

Our measurements were performed at the undulator beamline BW3 of the Hamburg Synchrotron Radiation Laboratory HASYLAB at DESY [9]. This undulator delivers intense photon beams of $\approx 10^{12}$ photons per second, monochromatized by a SX-700 monochromator. The experimental

\footnotetext{
*Present address: Physics Department, Medical Faculty, University of Rijeka, Braće Branchetta 20, 51000 Rijeka, Croatia.

†Electronic address: becker_u@fhi-berlin.mpg.de
}

setup is sketched in Fig. 1. The photon beam crosses an effusive beam of $\mathrm{C}_{60}$ molecules, provided by an oven resistively heated to $\approx 500{ }^{\circ} \mathrm{C}$. The size of the interaction region is determined by the intersection of the monochromatic photon beam (focal size $\approx 1 \mathrm{~mm}$ ) with the molecular beam (beam size $\approx 5 \mathrm{~mm}$ at about $5 \mathrm{~mm}$ above the outlet aperture). For the solid-state measurements, the $\mathrm{C}_{60}$ vapor was collected on a stainless-copper plate that could be moved into the interaction region after termination of the gas-phase measurement. Outgoing electrons are detected in time-of-flight (TOF) electron spectrometers at two different angles with respect to the electric vector of the ionizing radiation in order to derive the partial cross sections and the angular distribution asymmetry parameters.

For randomly oriented molecules in the gas phase, the differential cross section is characterized by the angular distribution asymmetry parameter $\beta[10]$ :

$$
\frac{d \sigma}{d \Omega}=\frac{\sigma(h \nu)}{4 \pi}\left[1+\beta(h \nu) P_{2}(\cos \theta)\right] .
$$

Here $\sigma$ is the partial photoionization cross section, $\Omega$ is the solid angle, $\theta$ is the angle between the electric vector of the

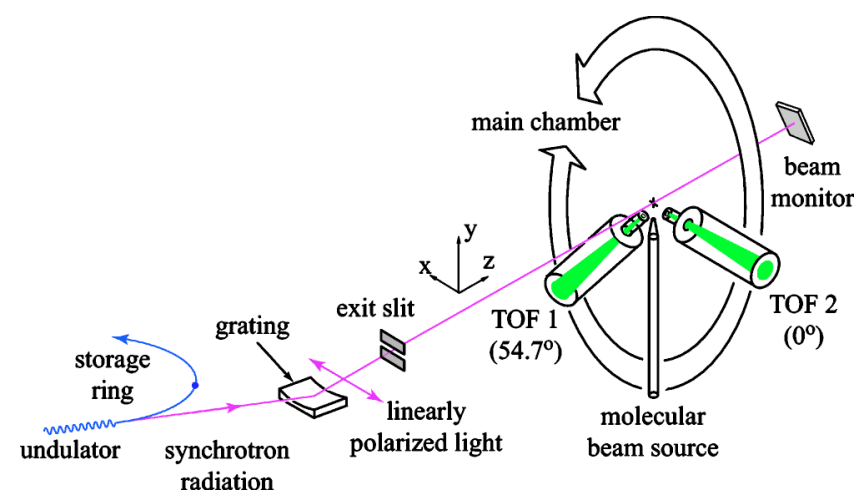

FIG. 1. Scheme of the experimental setup consisting of the rotatable electron time-of-flight spectrometers and an evaporative molecular beam source. 


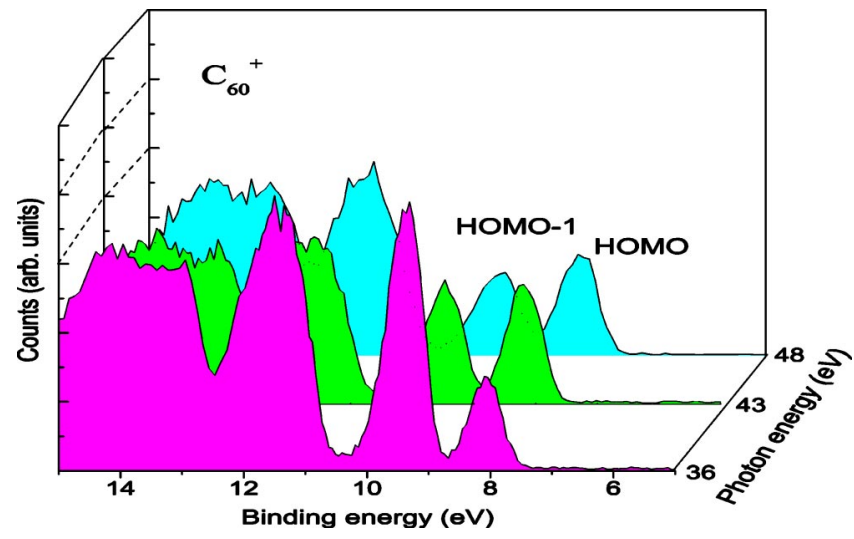

FIG. 2. The electron yield at the magic angle $\left(54.7^{\circ}\right)$, which is proportioned to the partial cross section, as a function of the binding energy for three different photon energies.

photon beam and the direction of the outgoing electron, and $P_{2}(\cos \theta)$ is the Legendre polynomial of second degree. At the so-called "magic angle" $\theta=54.7^{\circ}$, this polynomial becomes zero and the differential partial cross section becomes proportional to the partial cross section. Measurement at an additional angle determines $\beta$. In our setup, the two detectors were positioned at the "magic angle" and at $0^{\circ}$.

The transmission of the analyzers was determined using neon calibration spectra covering the energy region of interest and normalizing the $\mathrm{Ne} 2 p$ and $2 s$ lines with the known cross sections and angular distributions [11]. Additionally, the $\mathrm{C}_{60}$ spectra are normalized to the beamline flux profile, which was determined from the neon data and the photocurrent measured on a gold mesh placed in the beamline in front of the main chamber.

\section{RESULTS}

Typical gas-phase spectra for different photon energies are shown in Fig. 2. The experimental valence spectrum is characterized by a small number of distinct photoionization lines, which is consistent with the predicted high symmetry of the molecule [12] and the large degeneracy of the corresponding orbital levels. The first photoelectron line corresponds to the ionization from a single orbital of $h_{u}-\pi_{5}$ symmetry (HOMO) [5]. The second photoelectron line (HOMO-1) corresponds to the ionization from two different orbitals of $h_{g}-\pi_{4}$ and $g_{g}-\pi_{4}$ symmetry, which are nearly degenerate. Other photoelectron lines are broader and not well resolved. The results show systematic variations in the line intensities as a function of the photon energy. Each maximum of an odd state (HOMO) is matched by a minimum in intensity of an even state (HOMO-1). These oscillations are well known and have been the subject of several investigations with simplified theoretical models [13-15] and the same behavior is actually measured both in the gas phase $[16,17]$ and the solid state [18]. Benning et al. [18] first observed these variations for condensed phase $\mathrm{C}_{60}$. They showed relative intensity modulations of these features with photon energy up to $\approx 100 \mathrm{eV}$ and interpreted these effects in terms of final-state symmetries and parity-selection rules.
These intensity modulations provide evidence for the existence of high-lying final states that are not plane-wave-like. It is natural to expect that the variations in the intensities of these two photolines should be strongly affected by the special geometrical structure of $\mathrm{C}_{60}$, which is quite peculiar in nature: the carbon atoms form a cagelike structure enclosing a large cavity, and the high point group symmetry $I_{\mathrm{h}}$ makes all $\mathrm{C}$ atoms equivalent. It turned out [13] that the oscillations originate from the interference of photoelectron waves emanating from the 60 carbon atoms, i.e., the multicentered photoemission with the phase difference of each wave. Furthermore, the analytical calculation with a simplified conductingshell model [15] revealed that the spherical structure of the $\mathrm{C}_{60}$ molecule and its large radius are connected with the oscillations. In this paper we will compare our results for free molecules with corresponding measurements of condensed phase $\mathrm{C}_{60}$. The photoelectron spectra of the condensed-phase $\mathrm{C}_{60}$ show sharp, well-defined features HOMO and HOMO-1. The comparison of these spectra with a gas-phase spectrum reveals a close similarity, indicating the weakness of the interaction (which is of the van der Waals type) between $\mathrm{C}_{60}$ molecules in the molecular solid [19]. However, the relative intensities of the valence photoemission lines of solid and gaseous $\mathrm{C}_{60}$ are different, most likely because of inelastic scattering effects and differences in the corresponding transition matrix elements.

The branching ratio of the two outermost molecular orbitals HOMO and HOMO-1 for the condensed phase is displayed in Fig. 3 together with different theoretical curves. It is independent of the photon flux and target density variations. The photon energy was changed from near threshold up to the carbon $K$ edge with $1-\mathrm{eV}$ increments. These new measurements of the partial cross-section data over a large energy range made it possible to analyze the observed oscillations in terms of the structural information of the fullerene.

The Fourier-transformed cross-section data (Fig. 4) can be used to determine the radius of the fullerene $(R)$ and the thickness of the delocalized electron cloud $(\Delta)$. One has to keep in mind that these geometrical properties of the $\mathrm{C}_{60}$ molecule are not directly displayed by the peak positions in the plotted Fourier spectrum; they correspond rather to the asymptotic frequencies $f_{\ll}^{-1}$ being proportional to the energywindow-dependent spectral frequency $f^{-1}$. The photoelectron cutoff energy of $280 \mathrm{eV}$ used in this study corresponds to a proportionality factor of 1.085 , giving rise to a radius $R$ $=3.55 \AA$, in good agreement with the electron scattering results of Vos et al. [20] and most theoretical calculations. It is interesting to note that the agreement with the jellium calculation is improved compared to the former less densely measured data set, particularly concerning the low-frequency beating peak.

Figure 5 shows partial cross sections of $\mathrm{C}_{60}$ 's two outermost orbitals for the gas phase. The photon energy was increased in small steps of $0.1 \mathrm{eV}$ in the photon energy range from 19 to $50 \mathrm{eV}$, and in larger steps of $1 \mathrm{eV}$ from 50 to 70 $\mathrm{eV}$. The experimental results are compared with two different theoretical calculations. The first theoretical $a b$ initio calculation [21] employed a convergent one-center expansion and a local density approximation (LDA) Hamiltonian, which explicitly include the nonspherical nature of the potential. The 


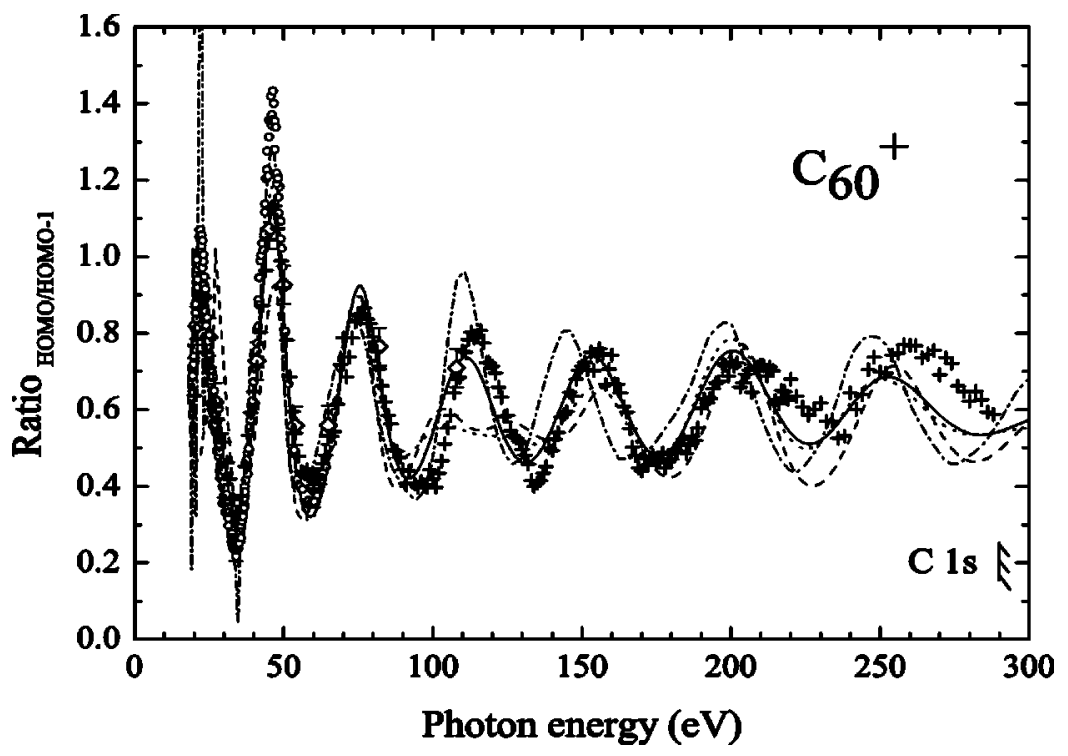

FIG. 3. Partial cross-section ratio $\mathrm{HOMO} /$ HOMO-1 for gas and solid $\mathrm{C}_{60}$ for different photon energies. The lines show the results of different theoretical models: theoretical curves with a jellium model (-----) and with LDA calculations (-.--); semiempirical curves with a shell model $(\cdots)$ and with a shell+cage model (-). The experimental data of Ref. [17] are shown by open rhombs, whereas the data of the present work are given by open circles for the gas-phase data and crosses for the solid-state measurements.

second theory uses Hartree-Fock (HF) molecular orbitals and solves the coupled scattering equations for the ejected electron in the field of the molecular ion within a polyatomic Schwinger variational method [22]. The general behavior of the partial cross section is very well described by the theory. Also, the quantitative agreement is quite satisfactory considering the scatter of the experimental data points. At low energies the photoionization cross section is dominated by the well-known plasmon peak resulting from collective electron behavior. For higher energies the ionization cross section exhibits oscillations with a frequency related to the diameter of the cluster superposed on the exponential decay. The only discrepancy between theory and experiment is the predicted existence of the pronounced resonant structures in the partial cross sections. Both theories show such structures in their calculations, found, however, at different positions, while no sharp structures are found in the experimental data. Because of the present measurement it seems reasonable to assume that the resonances in the partial cross sections are probably quenched by the vibrations of the molecule. The complex resonant wave functions of the resonantly excited states are

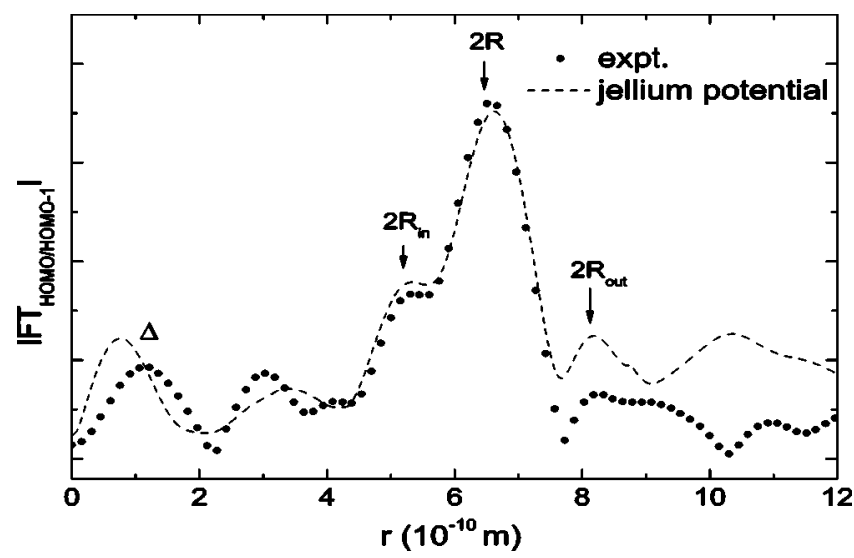

FIG. 4. Fourier transform of the cross-section ratio in Fig. 3 for the experimental data for the solid state (filled circles) and the LDA calculation using a spherical jellium potential (dashed line). in delicate balance between stabilization and decay forced by the different vibrational modes, such as the breathing mode of $\mathrm{C}_{60}$ [12]. In most cases the excited states are probably
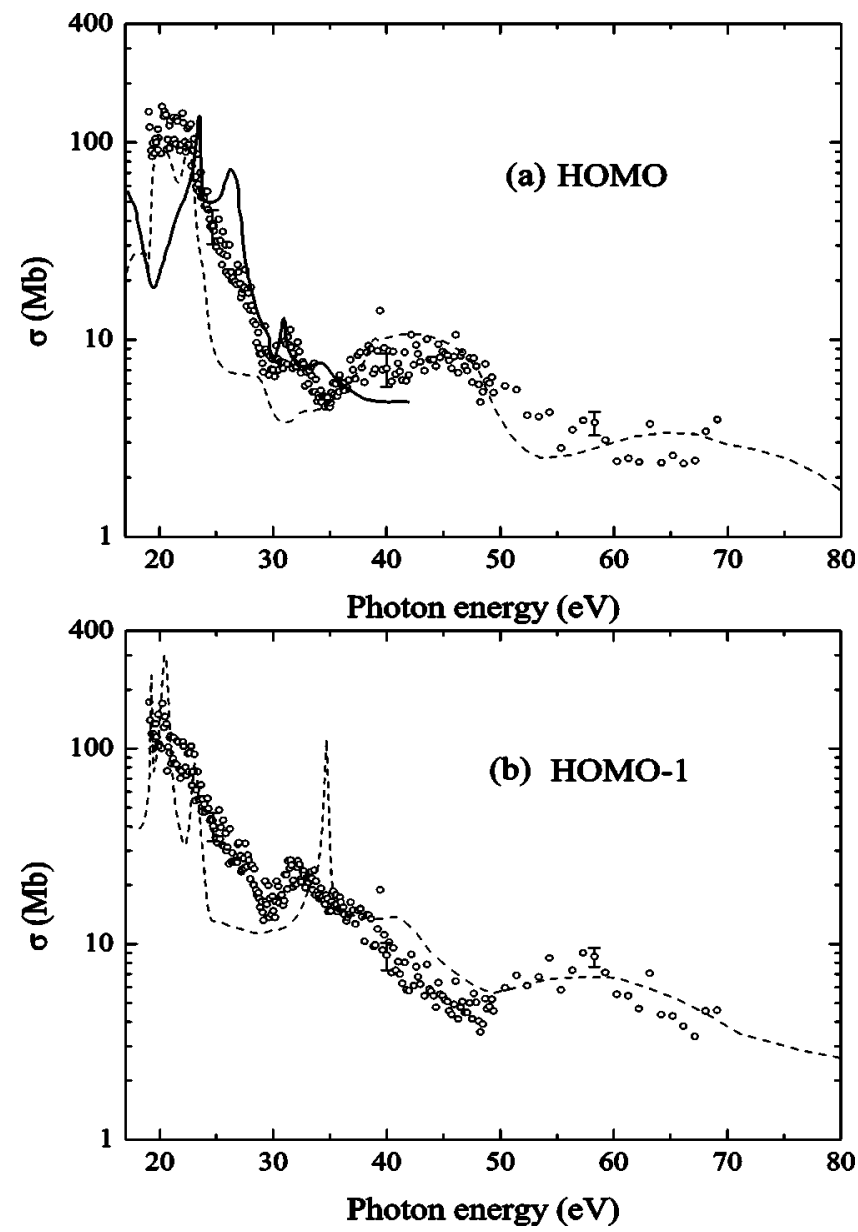

FIG. 5. Absolute cross sections for the two outermost orbitals of $\mathrm{C}_{60}$ (a) HOMO and (b) HOMO-1. The experimental points are shown by open circles. The theoretical curves are from Ref. [21] (dashed line) and Ref. [22] (solid line), respectively. 

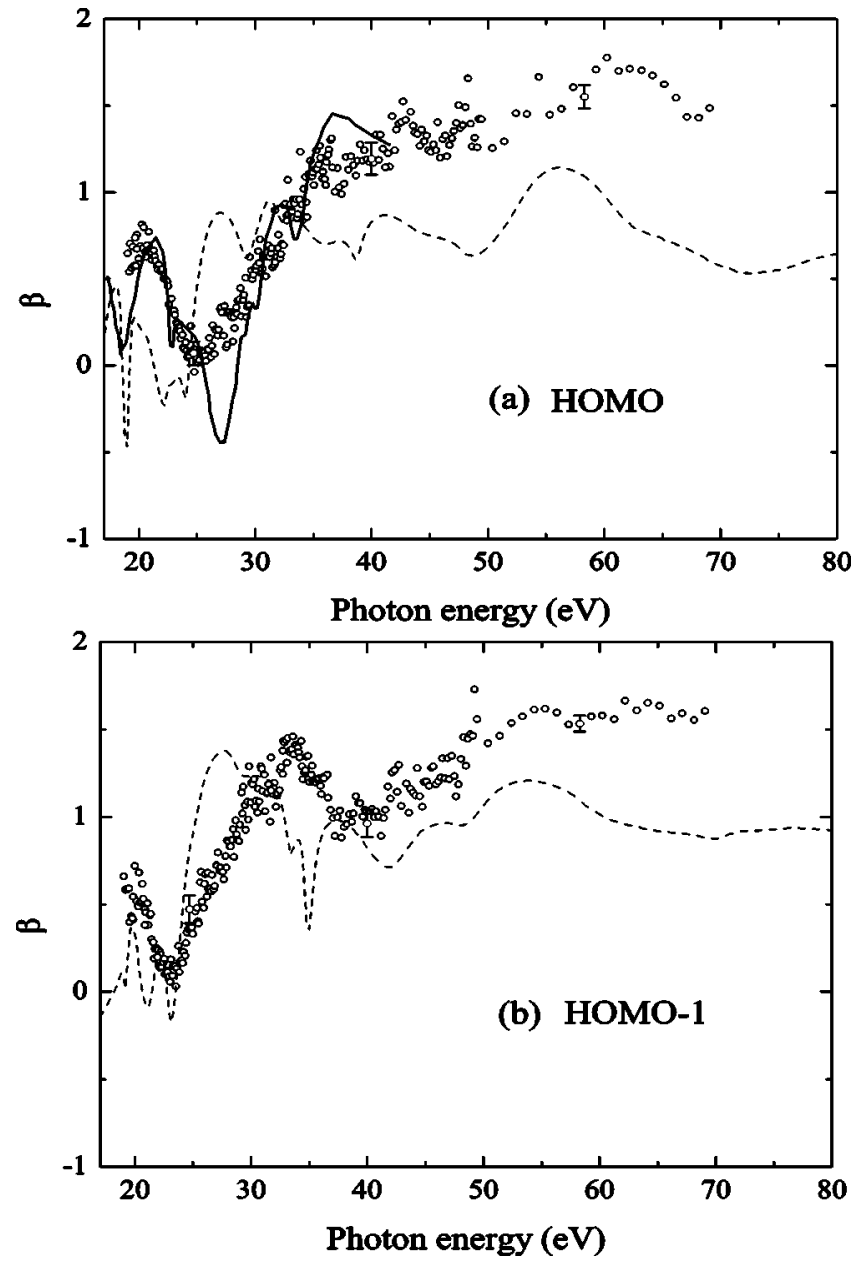

FIG. 6. The angular distribution asymmetry parameter $\beta$ for the two outermost orbitals of $\mathrm{C}_{60}$ (a) HOMO and (b) HOMO-1 as a function of the photon energy. The figure shows experimental results (open circles) and theoretical curves from Ref. [22] (solid line) and Ref. [23] (dashed line), respectively.

unstable and therefore do not appear in the partial cross section.

We also determined the angular distribution asymmetry parameter, $\beta$, of the HOMO and HOMO- 1 for the same photon energies (Fig. 6). In atomic and molecular photoionization, the $\beta$ parameter [10] reflects the photoelectron's angular momentum composition $[24,25]$. The angular momentum may be influenced by scattering of the photoelectron on other atoms in the molecule, giving rise to additional components and shifts in their relative phases. This phenomenon is in particular pronounced in shape resonances in molecular photoionization [26]. The theoretical curves for the $\beta$ values are very structured, especially in the low-energy region, while the experimental results show much smoother and wider structures. This is in accordance with the interpretation of the cross section oscillations as a quantum state effect of a three-dimensional box potential with no significant effect on the phase of the outgoing photoelectron wave. For both HOMO and HOMO-1, the anisotropy parameters $\beta$ change dramatically from zero to positive values over a very narrow photon energy range. They are both increasing after a minimum at about $25 \mathrm{eV}$, although this is shifted by a few eV with respect to each other. This behavior reflects the effect that the ejected electrons are emitted with increasing kinetic energy away from the isotropic distribution more towards the direction of the electric field of the light. The agreement between theory and experiment is, concerning this general trend, also satisfactory for the $\beta$ values, however, to a lower extent than for the partial cross sections. Even in the nonresonant regime the different theories deviate in the $\beta$ values from each other up to about half of a unit.

\section{SUMMARY}

In conclusion, we presented a study of the electronic structure and electron correlation effects in gas-phase and solid $\mathrm{C}_{60}$ molecules. The direct comparison of the spectra taken in both phases reveals a close similarity, indicating that solid-state interactions play only a minor role in this material, and electron correlation effects are predominantly intramolecular. For both $\sigma$ and $\beta$, the general trend, as well as the broad oscillatory features, are adequately described by theory, but the predicted pronounced resonance structures do not appear.

\section{ACKNOWLEDGMENTS}

This work was supported by the Bundesminister für Bildung und Forschung (BMBF) and in part by the Deutsche Forschungsgemeinschaft (DFG). The authors thank Piero Decleva for providing us unpublished data and many helpful discussions.
[1] H. W. Kroto, J. R. Heath, S. C. O'Brien, R. F. Curl, and R. E. Smalley, Nature (London) 318, 162 (1985).

[2] W. Krätschmer, L. D. Lamb, K. Fostiropoulos, and D. R. Huffman, Nature (London) 347, 354 (1990).

[3] A. Oshiyama, S. Saito, N. Hamada, and Y. Miyamoto, J. Phys. Chem. Solids 53, 1457 (1992).

[4] J. L. Martins, N. Troullier, and J. H. Weaver, Chem. Phys. Lett. 180, 457 (1991).

[5] R. C. Haddon, L. E. Brus, and K. Raghavachari, Chem. Phys.
Lett. 125, 459 (1986).

[6] M. Ozaki and A. Takahashi, Chem. Phys. Lett. 127, 242 (1986).

[7] R. Saito, G. Dresselhaus, and M. S. Dresselhaus, Phys. Rev. B 46, 9906 (1992).

[8] U. Becker and D. Shirley, VUV and Soft X-Ray Photoionization Studies (Plenum Press, New York, 1996).

[9] C. U. S. Larsson, A. Beutler, O. Björneholm, F. Federmann, U. Hahn, A. Rieck, S. Verbin, and T. Möller, Nucl. Instrum. 
Methods Phys. Res. A 337, 603 (1994).

[10] C. N. Yang, Phys. Rev. 74, 764 (1948).

[11] F. Wuilleumier and M. O. Krause, J. Electron Spectrosc. Relat. Phenom. 15, 15 (1979).

[12] G. Gensterblum, J. Electron Spectrosc. Relat. Phenom. 81, 101 (1996).

[13] S. Hasegawa, T. Miyamae, K. Yakushi, H. Inokuchi, K. Seki, and N. Ueno, Phys. Rev. B 58, 4927 (1998).

[14] Y. B. Xu, M. Q. Tan, and U. Becker, Phys. Rev. Lett. 76, 3538 (1996).

[15] O. Frank and J. M. Rost, Chem. Phys. Lett. 271, 367 (1997).

[16] T. Liebsch, O. Plotzke, F. Heiser, U. Hergenhahn, O. Hemmers, R. Wehlitz, J. Viefhaus, B. Langer, S. B. Whitfield, and U. Becker, Phys. Rev. A 52, 457 (1995).

[17] A. Rüdel, R. Hentges, U. Becker, H. S. Chakraborty, M. E. Madjet, and J. M. Rost, Phys. Rev. Lett. 89, 125503 (2002).
[18] P. J. Benning, D. M. Poirier, N. Troullier, J. L. Martins, J. H. Weaver, R. E. Haufler, L. Chibante, and R. E. Smalley, Phys. Rev. B 44, 1962 (1991).

[19] S. Krummacher, M. Biermann, M. Neeb, A. Liebsch, and W. Eberhardt, Phys. Rev. B 48, 8424 (1993).

[20] M. Vos, S. A. Canney, I. E. McCarthy, S. Utteridge, M. T. Michalewicz, and E. Weigold, Phys. Rev. B 56, 1309 (1997).

[21] P. Decleva, S. Furlan, G. Fronzoni, and M. Stener, Chem. Phys. Lett. 348, 363 (2001).

[22] F. A. Gianturco and R. R. Lucchese, Phys. Rev. A 64, 032706 (2001).

[23] P. Decleva (private communication).

[24] J. Cooper and R. Zare, J. Chem. Phys. 48, 942 (1968).

[25] U. Fano and D. Dill, Phys. Rev. A 6, 185 (1972).

[26] J. Dehmer and D. Dill, Phys. Rev. Lett. 35, 213 (1975). 